\title{
Mexican Trypanosoma cruzi Stocks: Analysis of Minicircle kDNA Homologies by Cross-hybridization
}

\author{
Marie-France Bosseno, Bertha Espinoza*, Beatriz Sánchez*, \\ Simone Frédérique Brenière/ ${ }^{+}$
}

\author{
UMR CNRS/ORSTOM No. 9926, BP 5045, 34032 Montpellier Cédex 1, France *Universidad Nacional \\ Autónoma de México, Instituto de Investigaciones Biomédicas, AP 70228, CP 04510, México, D.F.
}

\begin{abstract}
Homologies of minicircle kDNA of 27 Mexican stocks were studied by cross-hybridization with four kDNA probes derived from three reference stocks belonging to groups Trypanosoma cruzi I (SO34 cl4 and Silvio) and T. cruzi II (MN) and one Mexican stock. High homologies were only observed with Silvio (six stocks) and Mexican probes (11 stocks). After 30 min exposure (low homology) additional stocks were recognized with SO34 cl4 (three stocks) and Silvio (six stocks) probes; with the Mexican probe only five stocks remained non-reactive. All the stocks were typed by isoenzyme (16 loci) and Mexican stocks belonged to T. cruzi I. Hybridization patterns were not strictly correlated with the observed clustering and cross-hybridization of kDNA minicircles is not available to distinct Mexican stocks.
\end{abstract}

Key words: Trypanosoma cruzi - Mexico - kDNA minicircle

Homologies of the hyper variable regions of minicircle of kinetoplast-DNA (HVRm), obtained by polymerase chain reaction (PCR) from Mexican Trypanosoma cruzi stocks, were analyzed by hybridization with four probes: three kDNA probes were derived from reference stocks, SO34 cl4, MN, belonging to clonet 20 and 39 respectively (Brenière et al. 1998a,b), Silvio belonging to zymodeme I defined by Miles et al. (1980), and one probe from a Mexican stock isolated in Morelos state from Triatoma sp. (Mor 12 stock). T. cruzi taxon is divided in two major highly polymorphic lineages (T. cruzi I and T. cruzi II) as evidenced by molecular and genetic analysis (Tibayrenc 1995, 1998, Souto et al. 1996, Luquetti et al. 1999). Clonet 20 and ZI parasites belong to T. cruzi I and clonet 39 to T. cruzi II. The 27 Mexican stocks were from different states and isolated from different vectors, human and sylvatic mammals. Seven other stocks from South America

\footnotetext{
This work was supported by a grant from the "Institut de Recherche pour le Développement", DGAPA IN 224 798 UNAM and Conacyt 27951M.

${ }^{+}$Corresponding author. Fax: +525- 622 3369. E-mail breniere@servidor.unam. at Instituto de Investigaciones Biomédicas, Ciudad Universitaria, UNAM, Departamento de Inmunología, AP 70228, CP 04510, México D.F.

Received 12 July 1999

Accepted 23 February 2000
}

were used as controls. All the stocks were characterized by isoenzymes (16 loci) on cellulose acetate plates and a pairwise Jaccard's distance matrix was depicted in an unweighted pair-group method with arithmetic average (UPGMA) dendrogram. Among the stocks, 22 zymodemes were identified and distributed in the two groups of $T$. cruzi and all the Mexican stocks, belonged to T. cruzi I (Fig.1). Moreover, the great majority of the T. cruzi I Mexican stocks showed high genetic identity between them (Jaccard's distances < 0.20).

Probes were produced by PCR according to Brenière et al. (1992). Each probe was then hybridized using direct nucleic acid labeling and detection system (ECL, Amersham, Buckinghamshire, UK) with standardized HVRm PCR products of the studied stocks after their transfer to membranes by Southern blot. After overnight hybridization, the membranes were exposed successively during 1, 5 and $30 \mathrm{~min}$. Positive results after 1 and 5 min exposure were considered as reflecting high DNA homology and, low homology was considered when the hybridization was only positive after 30 min exposure (Fig. 2).

Figure 1 indicates which zymodemes hybridized with the different probes after 1 and 5 min exposure. As expected, clonet 39 probe gave a strong hybridization with only the homologous stock (MN) and one control stock belonging to the same zymodeme (Z20); indeed, the other stocks presented high genetic distances to clonet 39. Among 
the studied stocks belonging to $T$. cruzi I, an absence of strict correlation between hybridization patterns and sub-clustering was observed. Clonet 20 probe hybridized strongly with the homologous stock (SO34 cl4) and Cuica, a reference stock which pertains to the same zymodeme (Z3) but did not hybridize with the Mexican Sma 220 stock (Z3); after 30 min exposure, only three additional Mexican stocks presented a low DNA homology with this probe (Z44, JRA and H1 stocks). ZI probe presented a high DNA homology with itself and three Mexican stocks, all clustered together, but hybridized also with three other stocks (Z12, Z11 and Z10) which were not more related to $Z 1$ probe than others which did not hybridize; after 30 min exposure, six additional Mexican stocks showed hybridiza-

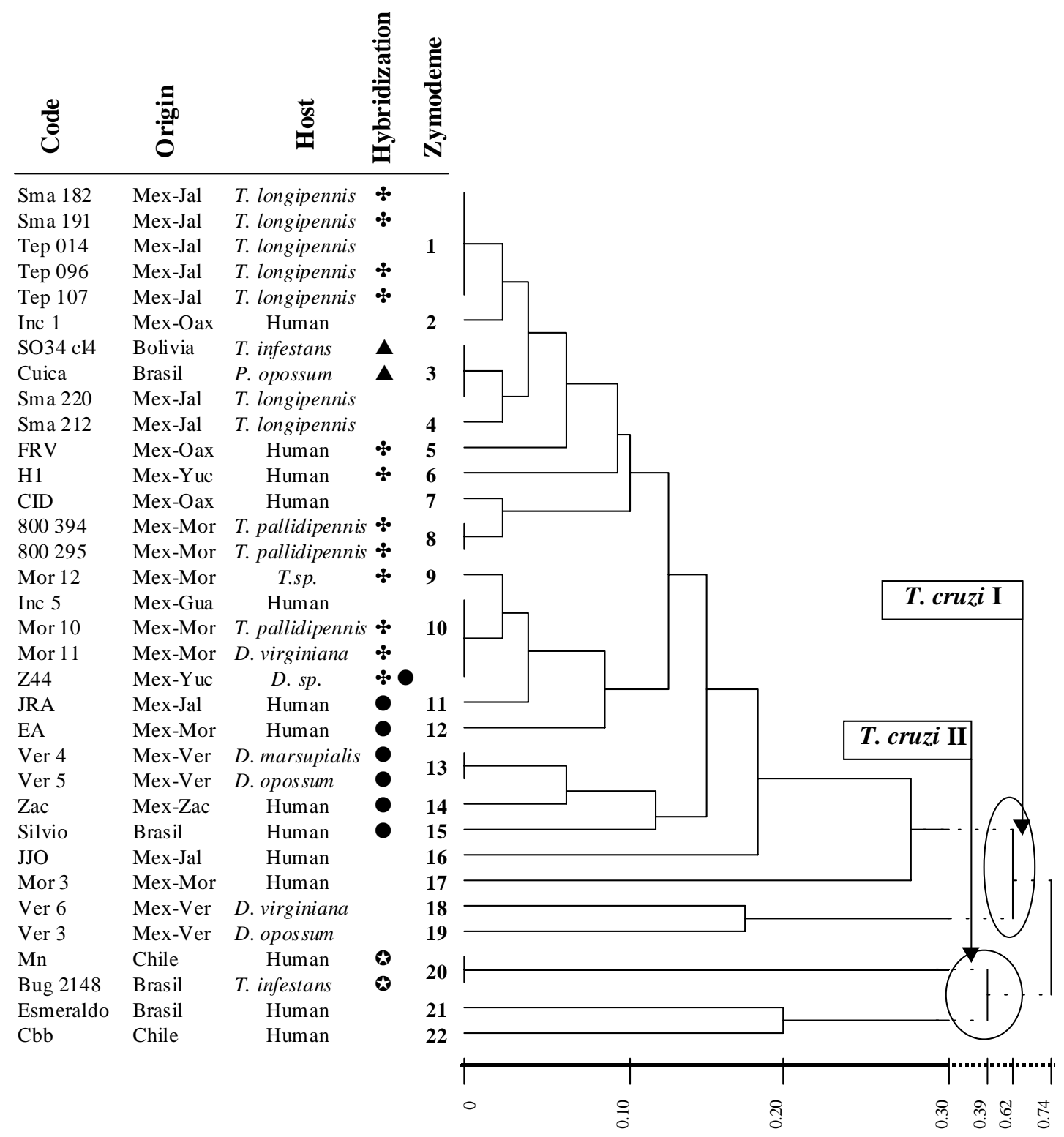

T.: Triatoma; P.: Philander; D.: Didelphis

Jaccard's distances

Fig. 1: dendrogram constructed using the unweighted pair group method with Jaccard's phenetic distance matrix based on a multi locus enzyme electrophoresis on 14 polymorphic loci among the 16 analysed. Geographical and host origin are indicated for each studied stock: Mex: Mexico; Mexican States - Gua: Guanajuato, Jal: Jalisco, Mor: Morelos, Oax: Oaxaca, Ver: Veracruz, Yuc: Yucatán, Zac: Zacatecas. Zymodeme numbering (Z1 to Z22) is according to the topology of the dendrogram. The stocks presenting a positive hybridization after 1 and $5 \mathrm{~min}$ of exposure are indicated for each probe: clonet 39: $\boldsymbol{\Theta}$, clonet 20: $\boldsymbol{\Delta}$, ZI: $\boldsymbol{\bullet}$, and Mexican: $\bullet$. 


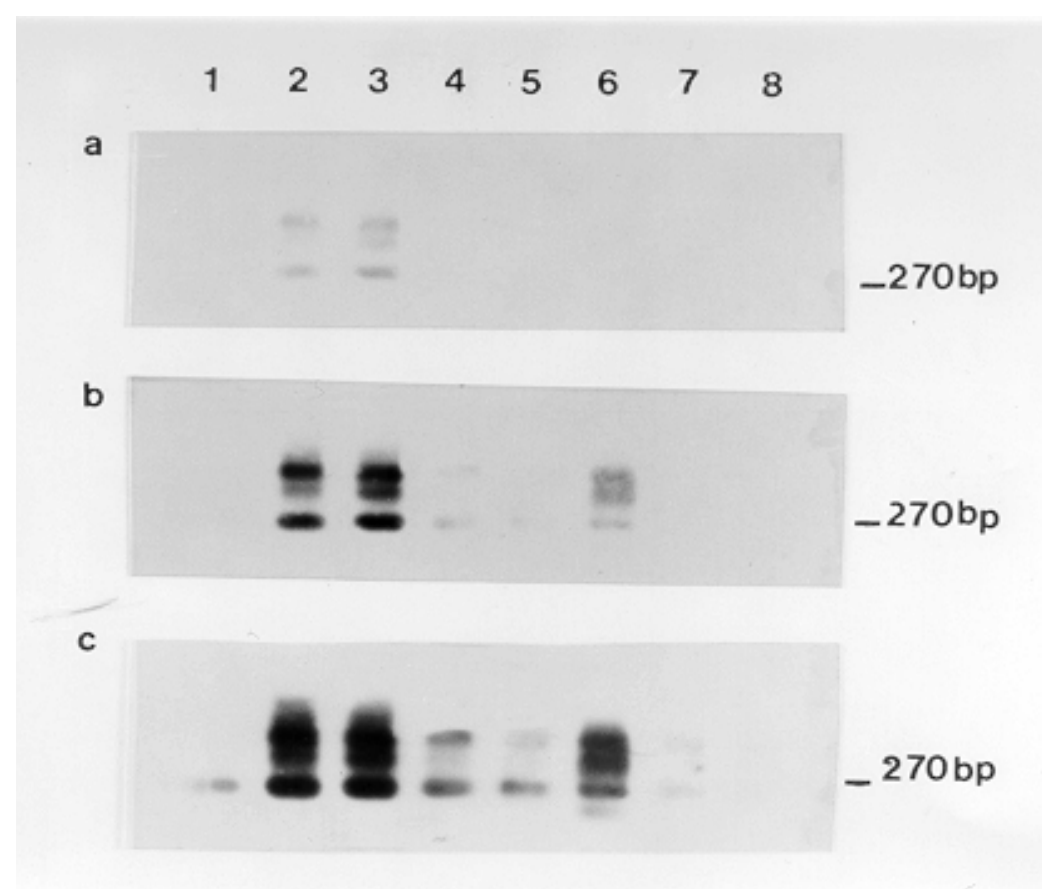

Fig. 2: hybridization of kDNA-PCR products obtained from Trypanosoma cruzi stock total DNA, with the Mexican kDNA probe (Mor 12 stock) after: (a) $1 \mathrm{~min}$, (b) $5 \mathrm{~min}$ and (c) $30 \mathrm{~min}$ exposures. Lanes 2, 3 and 6, Mexican stocks hybridized after 5 min exposure (high homology); lanes 1, 4, 5 and 7, Mexican stocks only hybridized after 30 min exposure (low homology); lane 8, a non-reactive Mexican stock

tion signal indicating low homology (Tep 014, Sma 220, Sma 212, CID, Mor 10 and Mor 11). The Mexican probe (Z9) had high DNA homology with 11 Mexican stocks (Fig. 1) which are not necessarily the most closely related zymodemes to this probe; after 30 min exposure, five Mexican stocks remained negative (Ver 3, Ver 6 and CID).

The absence of strict correlation between hybridization patterns and sub-clustering contrasted with studies showing that kDNA probes identified distinct monophyletic groups (Brenière et al. 1998a) but similar results were observed previously among Bolivian stocks isolated from $T$. sordida vector (Brenière et al. 1998b). The HVRm are highly heterogeneous in sequence (Morel et al. 1980, Avila et al. 1990) and the mechanisms which generate this diversity are unknown. Additional qualitative and quantitative studies of the different minicircle sequence classes would be necessary to best investigate the diversity and evolution of kDNA minicircles, particularly in Mexico where the vector species participate in sylvatic and domestic cycles. Finally, the minicircle sequences of $\mathrm{Z} 1$ and the Mexican probes had high homologies with $20 \%$ and $40 \%$ of the Mexican stocks respectively but these stocks were not clustered in separated sub- genetic groups. The current results do not support the use of kDNA probes to identify sub-genetic groups within Mexican stocks belonging to T. cruzi I as it was done previously in Bolivian field.

\section{ACKNOWLEDGMENT}

To Dr Felipe Lozano Kasten, Ezequiel Magallón Gástelum (Universidad de Guadalajara, Dept. of Public Health), Dr Janine Ramsey (Instituto Nacional de Salud Pública, Cuernavaca) and Dr Alejandro Cruz (Instituto de Biología, UNAM, Mexico) for their collaboration in the fieldwork to obtain the Mexican stocks.

\section{REFERENCES}

Avila H, Goncalves AM, Nehme NS, Morel CM, Simpson L 1990. Schizodeme analysis of Trypanosoma cruzi stocks from South and Central America by analysis of PCR-amplified minicircle variable region sequences. Mol Biochem Parasitol 42: 175-188.

Brenière SF, Bosseno MF, Revollo S, Rivera MT, Carlier Y, Tibayrenc M 1992. Direct identification of Typanosoma cruzi natural clones in vectors and mammalian hosts by polymerase chain reaction amplification. Am J Trop Med Hyg 46: 335-341.

Brenière SF, Bosseno MF, Telleria J, Bastrenta B, Yaksic N, Noireau F, Alcazar JL, Barnabé C, Wincker P, Tibayrenc M 1998a. Different behavior of two Try- 
panosoma cruzi major clones: transmission and circulation in Bolivian young patients. Exp Parasitol 89: 285-295.

Brenière SF, Morochi W, Bosseno MF, Ordoñez J, Gutierrez T, Vargas F, Yaksic N, Noireau F 1998b. Trypanosoma cruzi genotypes associated with domestic Triatoma sordida in Bolivia. Acta Trop 71: 269-283.

Miles MA, Lanham SM, DE Souza AA, Póvoa M 1980. Further enzymic characters of Trypanosoma cruzi and their evaluation for strain identification. Trans $R$ Soc Trop Med Hyg 74: 221-237.

Morel C, Chiari E, Camargo E, Mattei D, Romanha A, Simpson L 1980. Strains and clones of Trypanosoma cruzi can be characterized by restriction endonuclease fingerprinting of kinetoplast DNA minicircles. Proc Natl Acad Sci USA 77: 6810-6814.

Souto RP, Fernandes O, Macedo C, Campbell DA, Zingales B 1996. DNA markers define two major phylogenetic lineages of Trypanosoma cruzi. Mol Biochem Parasitol 83: 141-152.

Tibayrenc M 1995. Population genetics of parasitic protozoa and other microorganisms. Adv Parasitol 36: 47-115.

Tibayrenc M 1998. Genetic epidemiology of parasitic protozoa and other infectious agents: the need for and integrated approach. Int J Parasitol 28: 85-104. 